\title{
Immunohistochemical Demonstration of Acetaldehyde-modified Epitopes in Human Liver after Alcohol Consumption
}

\author{
Onni Niemelä," Tatu Juvonen, ${ }^{*}$ and Seppo Parkkila \\ ${ }^{*}$ Department of Clinical Chemistry, ${ }^{\ddagger}$ Surgery, and ${ }^{\S}$ Anatomy, Oulu University Central Hospital and \\ University of Oulu, Kajaanintie 50, SF-90220 Oulu, Finland
}

\begin{abstract}
Acetaldehyde, the toxic product of ethanol metabolism in the liver, covalently binds to a variety of proteins. Recent studies indicate that such binding can stimulate the production of antibodies against the acetaldehyde adducts. We raised rabbit antibodies which recognized various protein-acetaldehyde conjugates but not the corresponding control proteins. Such antibodies were used in immunohistochemical studies to find out whether acetaldehyde-generated epitopes can be detected from liver specimens of 13 human subjects with different degrees of alcohol consumption. While the specimens obtained from alcohol abusers $(n=4)$ and alcoholics $(n=3)$ exhibited marked positive staining for acetaldehyde adducts inside the hepatocytes in a granular uneven pattern, the control samples $(n=6)$ were almost devoid of immunoreactivity. In the alcohol abusers with an early stage of alcohol-induced liver damage, staining was detected exclusively around the central veins.

The data indicate that intracellular acetaldehyde adducts occur in the centrilobular region of the liver of individuals consuming excessive amounts of alcohol. Immunohistochemical detection of such adducts may prove to be of value in the early identification of alcohol abuse and in elucidating the mechanisms of alcohol-induced organ damage. (J. Clin. Invest. 1990. 87:1367-1374.) Key words: ethanol metabolism • alcoholism • acetaldehyde adducts
\end{abstract}

\section{Introduction}

A number of earlier in vitro studies have shown that acetaldehyde, the first metabolite of ethanol, can form covalent adducts with various proteins including hemoglobin, albumin, tubulin, and collagens (1-4). Previous studies have generally acknowledged that the liver, which is the primary site of ethanol metabolism, should also be rich in acetaldehyde-containing epitopes, and a number of adverse effects of ethanol have been attributed to adduct formation with liver proteins (5-7). It is believed to inhibit microtubule assembly and protein secretion (8-10), decreased enzyme activities (11) and increased protein catabolism (12). Recently, chronic alcohol consumption was demonstrated to lead to the appearance of serum antibodies which

Address correspondence and reprint requests to Dr. Onni Niemelä, University of Oulu, Department of Clinical Chemistry, Kajaanintie 50, SF-90220 Oulu, Finland.

Received for publication 7 May 1990 and in revised form $26 \mathrm{No}$ vember 1990.

J. Clin. Invest.

(c) The American Society for Clinical Investigation, Inc.

0021-9738/91/04/1367/08 \$2.00

Volume 87, April 1991, 1367-1374 recognize acetaldehyde-modified epitopes in proteins, indicating that acetaldehyde binding with proteins can also trigger immune responses (13-16). Since antibodies recognizing acetaldehyde adducts have also been successfully raised in laboratory animals by immunization with acetaldehyde-protein condensates $(13,17,18)$, attempts have been further made towards antibody-based detection of acetaldehyde condensates as potentially specific biological markers of excessive alcohol consumption $(17,19)$.

In recent experiments with rats fed alcohol chronically, Lin and co-workers $(18,20,21)$ detected a cytosolic $37-\mathrm{kD}$ liver protein-acetaldehyde adduct by an immunoblot technique using antibodies raised against hemocyanin-acetaldehyde. By contrast, Behrens et al. (22) found in similar experiments that cytochrome P450IIEl from hepatic microsomes of ethanol fed rats is a selective target of ethylation.

In this work we report the first immunohistochemical studies on acetaldehyde-modified epitopes in human liver based on rabbit polyclonal antibodies against the acetaldehyde adducts. These findings clarify the extent and localization of acetaldehyde binding with cellular components in the liver of individuals consuming ethanol.

\section{Methods}

Patients. Subjects with different degrees of alcohol consumption ( $n$ $=13$ ) were selected from patients undergoing surgery at the Oulu University Central Hospital. The reasons for surgery in these individuals were as follows: symptomatic gallstone disease $(n=8)$, liver trauma ( $n$ $=1)$, acute pancreatitis $(n=1)$, oesophageal cancer $(n=1)$, peritonitis $(n=1)$, and liver cirrhosis $(n=1)$. In all of them a liver biopsy was performed for diagnostic purposes.

All patients were systematically interviewed about their alcohol consumption. The average self reported daily consumption was from 0 to $150 \mathrm{~g}$. We also retrospectively administered the nine question Malmö-modified Michigan Alcoholism Screening Test, which has been recently shown to have reasonably high specificity for excessive drinking and recommended for the assessment of alcohol consumption in predominantly binge drinking populations (23). A score of three or more "yes" answers was considered to indicate alcohol abuse (23). There were four alcohol abusers with a history of spree drinking consisting of repeated inebriations but with no clinical and either no or only minimal biochemical or histological signs of chronic alcohol use (Table I). Two of these (subjects 1 and 4) had no previous medical or social records of alcohol related hospital admissions, disorderly behaviour, or major impairment in occupational functioning. We also studied three obvious alcoholics with advanced liver disease and six control individuals with either no or only minimal consumption $(\leq 20$ $\mathrm{g}$ of ethanol per wk). Some of the control subjects had liver disease such as fatty liver due to obesity or reactive hepatitis, one of them had persistently elevated transaminases due to exposure to industrial solvents, and one was diabetic. The relevant clinical and biochemical characteristics of these subjects are summarized in Table $I$.

The biopsy specimens obtained during surgery were divided into two parts after removal. The major one was for routine histological 
examination, and the other one was either immediately processed for the immunohistochemical study $(n=11)$ or frozen in liquid nitrogen until analysis $(n=2)$. The procedure had the approval of the Ethical Committee of Oulu University Central Hospital and the study was carried out according to the provisions of the Declaration of Helsinki.

Preparation of protein-acetaldehyde conjugates. Bovine serum albumin and low density lipoprotein were products of Sigma Chemical Co. (St. Louis, Mo.). Bovine type III pN-collagen $(4,24)$ and human red cell protein $(1,13)$ were purified as previously described. Before the preparation of the acetaldehyde condensates with these proteins, each of them was first dissolved in PBS, pH 7.4. The bovine serum albumin and human red cell protein solutions were subsequently dialyzed against PBS (at concentrations of 2 and $20 \mathrm{mg} / \mathrm{ml}$, respectively), containing various concentrations of acetaldehyde and $10 \mathrm{mM}$ sodium cyanoborohydride (from Sigma Chemical Co.) as described elsewhere $(13,14)$. The acetaldehyde solutions in buffer were prepared by appropriate dilutions of stock solutions of $\left[{ }^{14} \mathrm{C}\right]$ acetaldehyde $(9.5 \mathrm{mCi} / \mathrm{mmol}$; New England Nuclear, Boston, MA) with unlabeled acetaldehyde. The collagen and lipoprotein adducts were prepared by incubating these proteins $(1 \mathrm{mg} / \mathrm{ml}$ in PBS) with $100 \mu \mathrm{M}$ acetaldehyde and $1 \mathrm{mM}$ sodium cyanoborohydride for $5 \mathrm{~h}$ at $25^{\circ} \mathrm{C}$. The reaction mixtures were subsequently dialyzed against PBS to remove the unreacted acetaldehyde. All the above protein solutions were stored in aliquots at $-70^{\circ} \mathrm{C}$ until used.

Preparation of acetaldehyde adduct antibodies and immunoassays. Polyclonal anti-acetaldehyde adduct antibodies were raised in rabbits by subcutaneous injections of acetaldehyde-bovine serum albumin conjugate $(1 \mathrm{mg})$ prepared as above by $1 \mathrm{mM}$ acetaldehyde. Three booster injections were given with $500 \mu \mathrm{g}$ of the conjugate at 3-wk intervals. The animals were bled at 2-wk intervals after the second immunization and the anti-acetaldehyde adduct serum was cross-adsorbed on human plasma protein-acetaldehyde conjugate linked to Sepharose 4B (Pharmacia Fine Chemicals, Uppsala, Sweden) (13).

The antisera were subsequently tested for specificity in ELISA using $5 \mu \mathrm{g}$ (in $100 \mu \mathrm{l}$ PBS) of various protein-acetaldehyde conjugates to coat the wells (Titertek Flow laboratories, McLean, VA). Gelatin ( $0.2 \%$ ) was used to block nonspecific binding sites. After allowing the antisera to react with the acetaldehyde conjugates and the corresponding control proteins for $75 \mathrm{~min}$ at $37^{\circ} \mathrm{C}$, the antigen-antibody complexes were demonstrated using $\beta$-galactosidase-linked goat anti-rabbit immunoglobulin (Zymed Laboratories, San Francisco, CA) and the $O$-nitrophenyl- $\beta$-D-galactopyranoside color substrate. The optical densities were read at $405 \mathrm{~nm}$ by the Titertek Multiscan plate reader.

Immunohistochemical procedures. Specimens of human liver were fixed in Carnoy fluid for $12 \mathrm{~h}$ at $4^{\circ} \mathrm{C}$, dehydrated, and embedded in paraffin under a vacuum at $58^{\circ} \mathrm{C} .2-\mu \mathrm{m}$ sections were stained for acetaldehyde adducts by the peroxidase-antiperoxidase (PAP) ${ }^{1}$ complex method with slight modifications $(25,26)$. In essence, the steps in the PAP staining were as follows: (a) deparaffinization of the tissue sections; $(b)$ pretreatment of the sections with $3 \% \mathrm{H}_{2} \mathrm{O}_{2}$ for 5 min and rinsing in PBS for $5 \mathrm{~min}$; (c) pretreatment with swine serum (Lihapolar Co., Oulu, Finland) for $\mathbf{4 0}$ min to block unspecific binding and rinsing in PBS; $(d)$ incubation for $1 \mathrm{~h}$ in $1 / 100$ dilution of the primary rabbit antiserum in 1\% BSA-PBS; $(e)$ treatment with swine serum for $40 \mathrm{~min}$ and rinsing in PBS; $(f)$ incubation for $1 \mathrm{~h}$ in swine anti-rabbit serum IgG (DAKOPATTS, Copenhagen, Denmark) diluted $1 / 10$ in $1 \%$ BSAPBS; $(g)$ treatment with swine serum for $5 \mathrm{~min} ;(h)$ incubation for 30 min in a 1/100 dilution of PAP complex (DAKOPATTS) in PBS; and (i) incubation for $5 \mathrm{~min}$ in diaminobenzidine tetrahydrochloride (DAB) $\left(9 \mathrm{mg}\right.$ DAB in $15 \mathrm{ml}$ PBS plus $10 \mu 130 \% \mathrm{H}_{2} \mathrm{O}_{2}$ ).

After incubation steps 4,6 , and 8 , the sections were washed in PBS 3 times for $10 \mathrm{~min}$. All incubations and washings were carried out at room temperature and the sections were finally mounted in Permount (Fisher Scientific Co., Fair Lawn, NJ). The stained sections were examined with a Leitz Aristoplan microscope and photographed on

1. Abbreviations used in this paper: PAP, peroxidase-antiperoxidase. black and white negative film (Agfapan, 25 ISO; E. Leitz, Inc., Rockleigh, NJ).

Measurements of circulating antibody titers against acetaldehyde adducts. For this purpose, microtiter plates (Titertek) were coated with human red cell protein-acetaldehyde conjugates and the corresponding control protein solutions (both $50 \mu \mathrm{g} / \mathrm{ml}$ in PBS) as presented elsewhere (14). Gelatin $(0.2 \%)$ was used to block nonspecific binding sites. The sera were serially diluted in PBS-Tween and allowed to react with the coated proteins. After incubation, $\beta$-galactosidase-linked goat antihuman immunoglobulin (Zymed Laboratories, San Francisco, CA) was used to detect antigen-antibody complexes. The optic densities were read at $405 \mathrm{~nm}$ and the mean of duplicate ELISA absorbance readings was calculated and used to determine the antibody titers. The titers were expressed as difference scores, which were calculated by subtracting the immunoassay $\left(\mathrm{OD}_{405}\right)$ value obtained in a reaction with the sample (1:10 dilution) and the unconjugated protein from the value obtained with the sample (1:10 dilution) and the protein-acetaldehyde conjugate, and classified as follows: $0, \leq 0.050 ;+, 0.051-0.150 ;++$, $0.151-0.300 ;+++, \geq 0.301$

\section{Results}

The specificity of the rabbit antibodies raised against the acetaldehyde adducts were tested on ELISAs against acetaldehyde condensates with both circulating and structural proteins. Fig. 1 demonstrates that the antibodies recognized hemoglobin-,

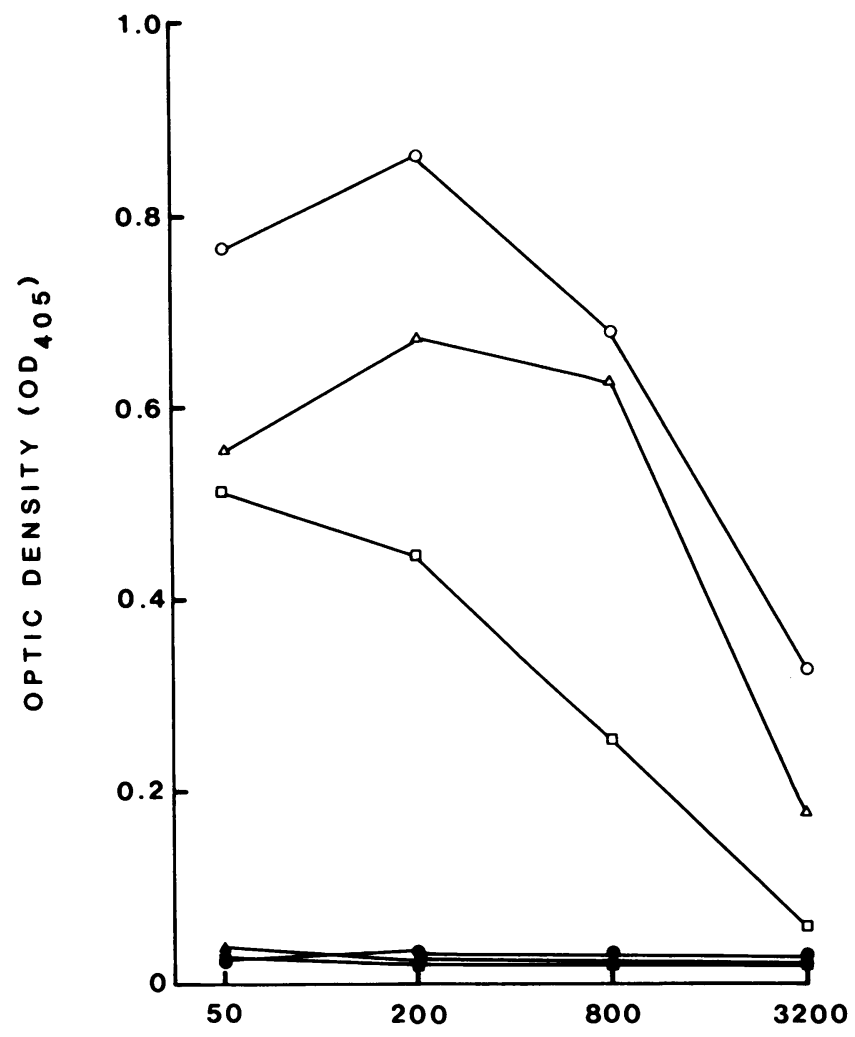

$1 /$ ANTIBODY DILUTION

Figure 1. ELISA of the rabbit anti-acetaldehyde adduct antibody tested against hemoglobin $(\square)$, lipoprotein $(\Delta)$, and collagen $(0)$ adducts prepared in vitro in the presence of $100 \mu \mathrm{M}$ acetaldehyde. The microtiter plates were coated with $5 \mu \mathrm{g}$ of the protein-acetaldehyde conjugates (open symbols) or the corresponding control proteins (closed symbols) with which the serial dilutions of the antibody were allowed to react. 
Table I. Clinical and Laboratory Characteristics of the Alcohol Abusers (1-4), Alcoholics (5-7), and Controls (8-13)

\begin{tabular}{|c|c|c|c|c|c|c|c|c|c|c|c|c|c|c|}
\hline $\begin{array}{l}\text { Subject } \\
\text { No. }\end{array}$ & Age & Sex & $\mathrm{H} / \mathrm{W}$ & Mm-Mast score & Ach-Ab & $\mathrm{Hb}$ & MCV & S-GT & S-ASAT & S-ALAT & S-AP & S-BIL & S-ALB & $\begin{array}{l}\text { Reason for } \\
\text { surgery }\end{array}$ \\
\hline & $y r$ & $M / F$ & $\mathrm{~cm} / \mathrm{kg}$ & & titer & g/liter & $f$ & \multicolumn{4}{|c|}{ U/liter } & umol/liter & g/liter & \\
\hline 1 & 56 & $\mathbf{M}$ & $175 / 57$ & 3 & ++ & 148 & 90 & 18 & 18 & 15 & 172 & 6 & 38 & CCE \\
\hline 2 & 49 & $\mathbf{M}$ & $165 / 60$ & 4 & + & 152 & 92 & 74 & 23 & 24 & 251 & 9 & 40 & $\mathrm{CCE}$ \\
\hline 3 & 32 & $\mathbf{M}$ & $175 / 63$ & 3 & ND & 106 & 90 & - & 59 & 44 & 83 & - & - & Liver trauma \\
\hline 4 & 55 & $\mathbf{M}$ & $173 / 50$ & 3 & ND & 143 & 100 & 30 & 18 & 10 & 170 & - & 33 & EC \\
\hline 5 & 51 & $\mathbf{M}$ & $172 / 80$ & 8 & ++ & 160 & 104 & 222 & 42 & 43 & 153 & 15 & 41 & CCE \\
\hline 6 & 60 & F & $155 / 50$ & 7 & ++ & 116 & 95 & 124 & 39 & 24 & 337 & 124 & 33 & MVB \\
\hline 7 & 31 & $\mathbf{M}$ & $175 / 72$ & 7 & ND & 98 & 100 & 60 & 19 & 30 & 209 & 7 & 21 & Acute pancreatitis \\
\hline 8 & 65 & $\mathrm{~F}$ & $163 / 83$ & 0 & 0 & 153 & 90 & 21 & 29 & 26 & 123 & 19 & 38 & $\mathrm{CCE}$ \\
\hline 9 & 29 & $\mathrm{~F}$ & $165 / 84$ & 0 & + & 138 & 87 & 34 & 24 & 50 & 162 & 6 & 36 & CCE \\
\hline 10 & 42 & $\mathbf{M}$ & $174 / 81$ & 0 & 0 & 135 & 80 & 30 & 40 & 70 & 100 & - & - & $\mathrm{CCE}$ \\
\hline 11 & 73 & $\mathrm{~F}$ & $150 / 59$ & 0 & 0 & 150 & 90 & 19 & 23 & 20 & 253 & 14 & 33 & CCE \\
\hline 12 & 61 & $\mathbf{M}$ & $185 / 100$ & 0 & ND & 109 & 84 & - & 27 & 25 & 248 & 18 & 31 & Peritonitis \\
\hline 13 & 40 & $\mathbf{F}$ & $165 / 61$ & 0 & ND & 136 & 90 & - & 15 & 8 & 105 & 15 & 31 & CCE \\
\hline
\end{tabular}

CCE, cholecystectomy; MVB, mesochaval vena bypass due to portal hypertension; EC, esophageal cancer; $\mathrm{H} / \mathrm{W}$, height/weight; $\mathrm{Mm}-\mathrm{Mast}$, Malmö-modified Michigan Alcoholism Screening Test; Ach-Ab, titer of circulating antibodies against acetaldehyde adducts; Hb, hemoglobin; $\mathrm{MCV}$, mean corpuscular volume; S, serum; GT, gamma glutamyl transpeptidase; ASAT, aspartate aminotransferase; ALAT, alanine aminotransferase; AP, alkaline phosphatase; BIL, bilirubin; and ALB, albumin. - , no labeling; + , weak labeling; ++, moderate labeling; +++, intense labeling.

lipoprotein-, and collagen-acetaldehyde adducts prepared in vitro at $100 \mu \mathrm{M}$ acetaldehyde, concentration which may exist in the hepatocyte as a result of alcohol consumption (27).

The liver biopsy specimens obtained from the control subjects and from those consuming alcohol were stained with the acetaldehyde adduct antibodies using the PAP-complex method. The subjects studied included four heavy drinkers, three alcoholics, and six controls either with or without liver disease. The relevant clinical and laboratory characteristics of all subjects are given in Table $I$. This table also gives the results of the measurements of the antibody titers against acetaldehyde adducts from eight subjects of whom serum samples were available. Elevated levels were observed from 2 of 2 alcoholics, 1 of 2 alcohol abusers, and 1 of 4 controls.

The overall distribution and intensity of staining for acetaldehyde adducts in the alcohol abusers and nonabusers is summarized in Table II. All the specimens $(n=7)$ obtained from the individuals with a history of excessive alcohol consumption showed positive intracellular staining for acetaldehyde adducts in the liver. A sample from a 57-y-old male heavy drinker with a history of spree drinking but no clinical or biochemical signs of chronic alcohol use, and with only slight signs of liver injury at the light microscopical examination is presented in Fig. 2. A rather intense peroxidase reaction can be seen inside the hepatocytes displaying an uneven granular pattern (Fig. $2 A$ ). Some of the nuclei were also found to be stained (Fig. $2 A$ ). The hepatocytes exhibiting immunoreactivity were detected primarily in the perivenous region (Fig. $2 B$ ), whereas the hepatocytes in the periportal region were almost devoid of immunoreactivity (Fig. 2 C). A similar pattern of strong positive centrilobular staining around the central vein was also observed from a 49-y-old male heavy drinker despite at least 2 -wk period of abstinence before surgery (Fig. 3).

In the alcoholic patients with advanced liver fibrosis and marked clinical signs of chronic alcoholism, a slightly different pattern of positive staining was seen (Table II). The number of immunostained hepatocytes in these cases appeared to be
Table II. Histological and Immunohistochemical Data on the Liver Specimens from Alcohol Abusers (1-4), Alcoholics (5-7), and Controls (8-13)

\begin{tabular}{|c|c|c|}
\hline $\begin{array}{l}\text { Subject } \\
\text { No. }\end{array}$ & Routine histology & Acetaldehyde adducts \\
\hline 1 & $\begin{array}{l}\text { Mild fatty deposition, } \\
\text { minimal portal fibrosis }\end{array}$ & $\begin{array}{l}+++, \text { most perivenous } \\
\text { regions }\end{array}$ \\
\hline 2 & $\begin{array}{l}\text { Mild fatty deposition, } \\
\text { minimal perivenular } \\
\text { fibrosis }\end{array}$ & $\begin{array}{l}+++, \text { most perivenous } \\
\text { regions }\end{array}$ \\
\hline 3 & Mild fatty deposition &,++ most perivenous regions \\
\hline 4 & Portal dilation, slight fibrosis &,++ most perivenous regions \\
\hline 5 & $\begin{array}{l}\text { Marked fatty deposition, } \\
\text { alcoholic fibrosis }\end{array}$ & $\begin{array}{c}+++, \text { widespread, most } \\
\text { perivenous regions }\end{array}$ \\
\hline 6 & Advanced liver cirrhosis & $\begin{array}{l}+ \text {, widespread, inside many } \\
\text { of the surviving } \\
\text { hepatocytes }\end{array}$ \\
\hline 7 & $\begin{array}{l}\text { Mild/moderate fatty change, } \\
\text { moderate fibrosis, } \\
\text { sinusoidal dilatation }\end{array}$ & + , perivenous regions \\
\hline 8 & Severe fatty change & - \\
\hline 9 & $\begin{array}{l}\text { Fatty change, reactive } \\
\text { hepatitis }\end{array}$ & - \\
\hline 10 & $\begin{array}{l}\text { Fatty change, regenerative } \\
\text { hepatocytes, lymphocyte } \\
\text { infiltrate in the periportal } \\
\text { tract }\end{array}$ & - \\
\hline 11 & Sinusoidal dilatation & + , occasional hepatocytes \\
\hline 12 & $\begin{array}{l}\text { Mild fatty change, } \\
\text { sinusoidal dilatation }\end{array}$ &,+ a few pericentral regions \\
\hline 13 & $\begin{array}{l}\text { Fatty change, a few } \\
\text { lymphocytes in the } \\
\text { periportal tract }\end{array}$ & - \\
\hline
\end{tabular}

- , no labeling; + , weak labeling; ++ , moderate labeling; +++ , intense labeling. 

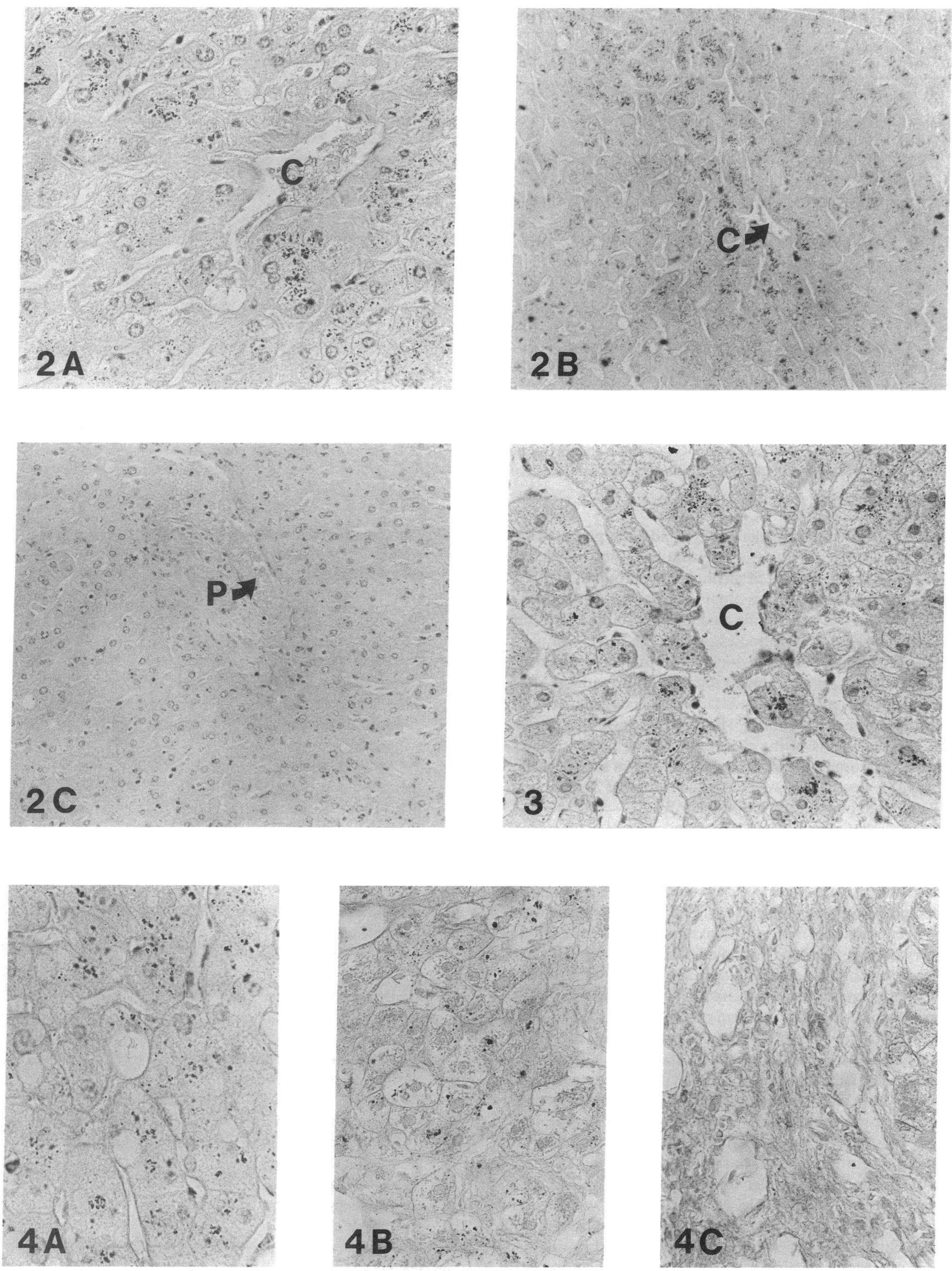
smaller than in the heavy drinkers with an earlier stage of alcoholic liver disease (Fig. 4, $A$ and $B$ ). Furthermore, the perivenular predominance of staining was no longer evident. The fibrous septa replacing the parenchyma were not stained (Fig. $4 C$ ).

The individuals serving as controls $(n=6)$ had a history of either no or only minimal alcohol consumption. Some of them presented liver histology abnormalities due to gallstone disease and obesity, such as fatty deposition or reactive hepatitis; one of them had persistently elevated transaminases due to apparent exposure to industrial solvents and one had diabetes. No specific staining for acetaldehyde adducts was observed (Fig. 5, $A-E$ ), except that a minimal reaction inside a few hepatocytes was seen from a sample of a 73-yr-old diabetic woman reporting no alcohol consumption (Fig. $5 \mathrm{D}$ ) and from a 61-yr-old schizophrenic man with prolonged use of chlorpromazine and alcohol consumption of $\sim 20 \mathrm{~g} / \mathrm{wk}$ (Fig. $5 E$ ). Occasionally, some of the nuclei were also stained in the control patients. Additional control sections involving incubations of the liver specimen with the preimmune serum did not produce any staining (Fig. $5 F$ ).

\section{Discussion}

These studies comprise the first demonstration of acetaldehyde-modified epitopes in the liver of human subjects consuming alcohol. The immunohistological demonstration by the PAP-complex method is based on rabbit polyclonal antibodies recognizing acetaldehyde adducts with both circulating and structural proteins.

Previous in vitro studies have demonstrated the formation of acetaldehyde adducts with several hepatic proteins and cell constituents (2-8). Acetaldehyde derived from oxidation of $\left[{ }^{14} \mathrm{C}\right]$ ethanol by rat liver slices has also been shown to bind to preformed hepatocyte proteins (28). Adduct formation in vivo has been implicated in the appearance of circulating antibodies (13-17) and cytotoxic complexes (29) which recognize acetaldehyde-modified proteins prepared in vitro. Any injurious effect of immune reactions against acetaldehyde-modified proteins should, however, be dependent not only on the antibody titers but also on the introduction of antigens into the liver, i.e., acetaldehyde adducts as created by prolonged ethanol ingestion $(14,30)$. The extent of antigen formation in the liver has, however, remained unclear.

These findings indicating that acetaldehyde adducts are intracellular in the liver of individuals consuming ethanol may suggest that to stimulate an immunologic cascade and hepatocyte damage the release of intracellular antigens should be a necessary precondition. The presence of intracellular adducts is in agreement with recent observations by Lin and co-workers $(18,20,21)$ as well as with Behrens et al. (22). It should be noted, however, that the former group of investigators reported only one, yet unidentified, cytosolic 37-kD acetaldehyde-modified polypeptide, while the latter found only a cytochrome P450IIE1 to be a selective target of modification. This may be due to differences in the actual acetaldehyde adducts involved in the antibody-based detection (20). Although there may also be a difference in the metabolic rates of ethanol in rat and man (31), the staining patterns observed in this study suggest that several targets of acetaldehyde adduct formation should exist in human liver. Consistent with this view, several immunoreactive adducts were very recently observed from microsomes and cytosol of mouse liver (32).

The finding that the acetaldehyde-modified epitopes in the liver are concentrated in the perivenous region in an early stage of alcohol-induced liver damage is notable, since this region is also the predominant site of precursor lesions in alcoholic liver (33). An ethanol inducible cytochrome P450IIE1, which catalyzes the oxidation of ethanol to acetaldehyde, can also be found predominantly in perivenular hepatocytes $(34,35)$. The lobular distribution of alcohol dehydrogenase, the primary enzyme of alcohol metabolism, has remained controversial (33). Nevertheless, some studies have indicated that it is also concentrated in zone 3 of the hepatic acinus $(36,37)$.

At this time it is not possible to identify the exact sites of modification and further studies involving immunoelectronmicroscopy are in progress to clarify the issue. The intracellular granular staining in the hepatocytes could represent both the cytosolic compartment and the mitochondria. The presence of immunoreactive material in the nuclei of the hepatocytes may be consistent with the observations that exocyclic amino groups of ribonucleosides and deoxyribonucleosides can bind acetaldehyde (38). Extracellularly, no staining was observed. The lack of staining in the fibrous tissue may suggest that the binding of acetaldehyde to components of connective tissue, which has been previously demonstrated in vitro and in cell culture, may result in the formation of molecules which in vivo do not get deposited in the tissue $(4,39)$.

Immunocytochemical detection of acetaldehyde-generated epitopes may prove to be of value in the assessment of heavy drinking and/or ethanol-induced tissue damage (19). A marked positive reaction in the liver of patients with neither apparent histological or clinical signs of alcoholic liver disease

\footnotetext{
Figure 2. Immunohistochemical localization of acetaldehyde-modified epitopes in a liver biopsy specimen from a 56-yr-old man (subject 1) reporting episodes of heavy drinking and a total consumption of usually less than $1,500 \mathrm{~g}$ of ethanol per month. Routine histological examination revealed mild fatty deposition and minimal fibrosis. A granular pattern of staining is seen inside the hepatocytes $(A)$ (original magnification, $350)$. The positive staining representing the acetaldehyde adducts is clearly restricted to the region around the terminal vein $(C)(B)($ magnification, 180), whereas no specific granular staining could be seen in the periportal tract $(P)(C)$ (magnification, 180). Figure 3. Immunohistochemical staining for acetaldehyde adducts from subject 2 with a history of heavy drinking. The total self reported consumption was usually less than $2,000 \mathrm{~g}$ per month. An early stage of alcohol-induced liver damage, i.e., perivenular fibrosis is present in the liver biopsy. A marked reaction for acetaldehyde adducts is also seen inside the hepatocytes around the terminal vein $(C)$. Original magnification, 350. Figure 4. Immunohistochemical localization of hepatic acetaldehyde adducts from the alcoholics reporting a consumption of at least 2,000 $\mathrm{g}$ of ethanol per month. A biopsy specimen from a 51-yr-old alcoholic man (subject 5) with alcoholic fibrosis and marked fatty deposits shows a moderate granular staining in the hepatocytes $(A)$ (original magnification, 450). A specimen from a 60-yr-old female alcoholic (subject 6) with advanced liver cirrhosis. A slight positive reaction is present in the morphologically-damaged hepatocytes $(B)$ (magnification, 350 ). Note also that the fibrous tissue in the liver is not stained $(C)$ (subject 6) (magnification, 350).
} 

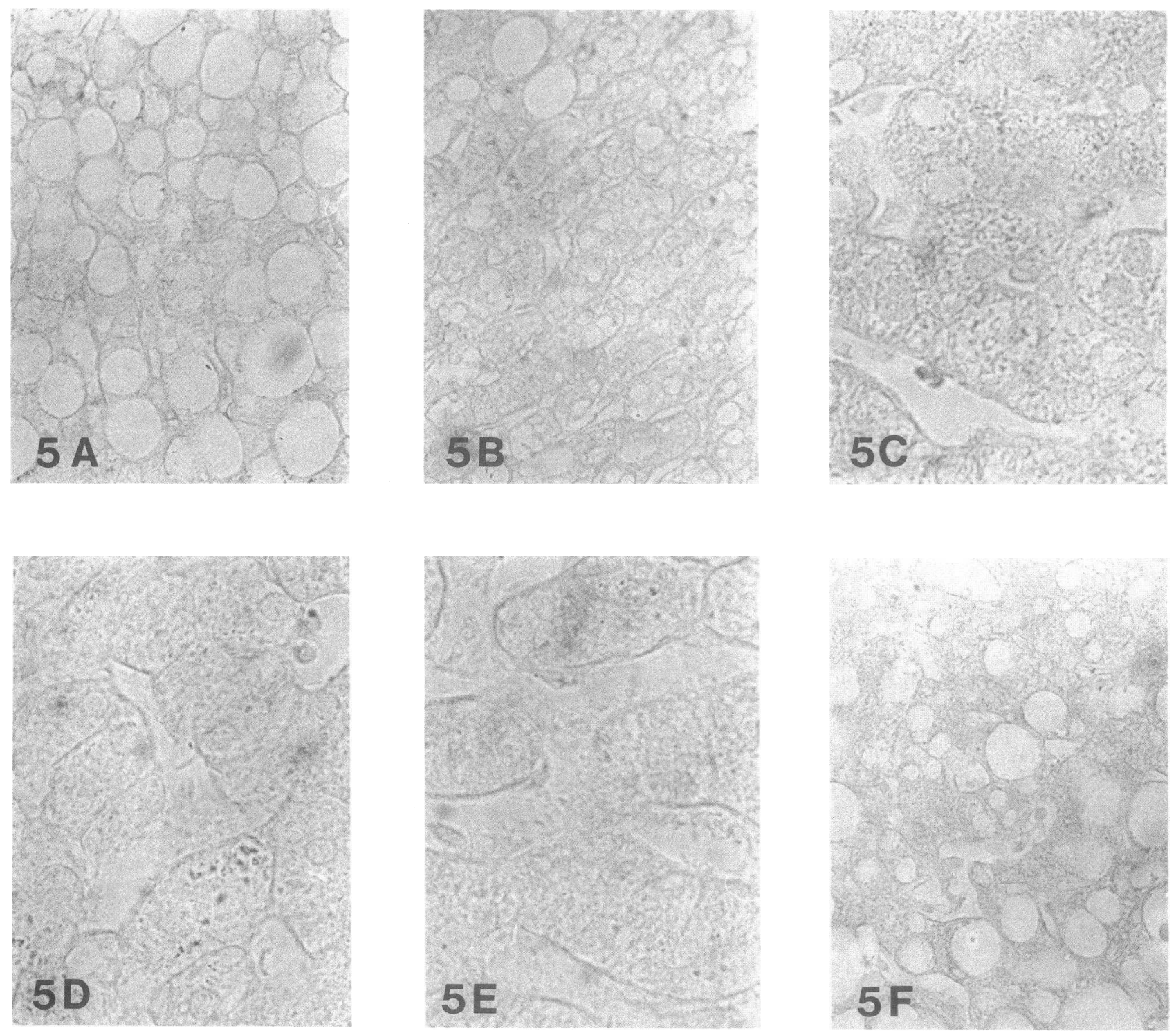

Figure 5. Immunohistochemical staining for acetaldehyde adducts in the control individuals. No specific immunostaining is seen from subjects with severe fatty liver due to obesity but with either no $(A)$ (subject 8 ) or only minimal, less than $20 \mathrm{~g}$ of ethanol per week $(B)$ (subject 9 ) alcohol consumption. Similarly, staining from a 42-yr-old man with a history of persistently elevated transaminases due to exposure to industrial solvents $(C)$ (subject 10 ) was also negative. A patient with diabetes, congestive cardiac failure, and no alcohol consumption $(D)$ (subject 11$)$ as well as a patient with schizophrenia, prolonged use of chlorpromazine medication, and $\sim 20 \mathrm{~g}$ of alcohol per week $(E)$ (subject 12$)$ had minimal reactions inside a few hepatocytes. Control stainings (sample from subject 5 ) with the preimmune serum showed no immunoreactivity $(F)$. Original magnifications: $A, B$, and $F \times 350 ; C-E \times 750$.

nor medical or social records of alcoholism appears promising. The finding that the immunostaining in the obvious alcoholics was actually not any higher than in the heavy drinkers may suggest a smaller number of sites available for antibody-based detection, a greater loss of acetaldehyde-altered hepatocyte proteins in advanced liver disease, or most likely that the alcoholics do not actually consume as much ethanol as the heavy drinkers due to a lower threshold.

In this study, all the samples from alcohol consumers produced a positive reaction. In the controls, two positive reactions were, however, also obtained. While in one case the reason for this remains obscure, in the other the use of chlorproma- zine medication may have potentiated the effect of small alcohol amounts and this individual could in fact be a true positive. Future studies should address the diagnostic potential of the immunocytochemical approach in larger materials involving different stages of alcohol-associated medical disorders as well as nonalcoholic liver diseases, which are sometimes known to result in elevated endogeneous acetaldehyde levels (40). The approach would be analogous to the detection of acetaldehyde condensates with circulating proteins in order to evaluate cumulative exposure to ethanol comparable to the measurements of glucosylated hemoglobin to estimate glucose control in diabetes $(17,19,41,42)$. 
The chemical nature of the acetaldehyde adduct involved in the antibody-based detection has not been clearly defined. Earlier studies have indicated that acetaldehyde selectively modifies the epsilon amino groups of lysine residues (43) yielding immunogenic derivatives upon stabilization with appropriate reducing agents $(13,44,45)$. Reaction of acetaldehyde with proteins may, however, also involve other amino acid residues including cysteine, valine and tyrosine $(1,5,46)$, and other types of adducts of which at least the acetaldehyde-tyrosine linkage, possibly involving a cyclic imidazolidinone, has been shown to be immunoreactive $(20,46)$. At this time we also cannot rule out the possibility that the antibodies would recognize other types of arrangements, such as cross-linking, which is known to occur in reactions with aldehydes and cellular components $(47,48)$. Various other types of posttranslational nonenzymatic modification of plasma and structural proteins may also exist in vivo, including glucosylation of diabetes and carbamylation of uremia. Some preliminary results indicate, however, that the epitopes generated through glucosylation or carbamylation should not cross-react significantly with the antibodies raised against the acetaldehyde-modified proteins ( $O$. Niemelä and Y. Israel, unpublished observations).

In conclusion, we have demonstrated, through immunohistochemistry, the existence and distribution of acetaldehydemodified epitopes in the liver of human subjects consuming alcohol. These findings may give new insights into the pathophysiological role of acetaldehyde adducts in creating the adverse effects of ethanol consumption in the liver. Immunohistochemical visualisation of acetaldehyde adducts may prove to be a useful tool to pay attention to harmful alcohol consumption before the patient begins to develop cirrhosis.

\section{Acknowledgments}

This study was supported by a grant from the Finnish Foundation for Alcohol Studies.

\section{References}

1. Stevens, V. J., W. F. Fantl, C. B. Newman, R. V. Sims, A. Cerami, and C. M. Peterson. 1981. Acetaldehyde adducts with hemoglobin. J. Clin. Invest. 67:364-369.

2. Tuma, D. J., T. M. Donohue, V. A. Medina, and M. F. Sorrell. 1983. Acetaldehyde adducts with proteins: binding of ${ }^{14} \mathrm{C}$-acetaldehyde to serum albumin. Arch. Biochem. Biophys. 220:239-246.

3. Jennett, R. B., M. F. Sorrell, A. Saffari-Fard, J. L. Ockner, and D. J. Tuma. 1989. Preferential covalent binding of acetaldehyde to the $\alpha$-chain of purified rat liver tubulin. Hepatology. 9:57-62.

4. Jukkola, A., and O. Niemelä. 1989. Covalent binding of acetaldehyde to type III collagen. Biochem. Biophys. Res. Commun. 159:163-169.

5. Sorrell, M. F., and D. J. Tuma. 1987. The functional implications of acetaldehyde binding to cell constituents. Ann. NY Acad. Sci. 492:51-62.

6. Nomura, F., and C. S. Lieber. 1981. Binding of acetaldehyde to rat live microsomes: enhancement after chronic alcohol consumption. Biochem. Biophys. Res. Commun. 100:131-137.

7. Donohue, T. M., D. J. Tuma, and M. F. Sorrell. 1983. Binding of metabolically derived acetaldehyde to hepatic proteins in vitro. Lab. Invest. 49:226-229.

8. Smith, S. L., R. B. Jennett, M. F. Sorrell, and D. J. Tuma. 1989. Acetaldehyde substoichiometrically inhibits bovine neurotubulin polymerization. J. Clin Invest. 84:337-341.

9. McKinnon, G., J. de Jersey, B. Shanley, and L. Ward. 1987. The reaction of acetaldehyde with brain microtubular proteins: formation of stable adducts and inhibition of polymerization. Neurosci. Lett. 79:163-168.

10. Baraona, E., M. A. Leo, S. A. Borowsky, and C. S. Lieber. 1977. Pathogenesis of alcohol-induced accumulation of protein in the liver. J. Clin. Invest. 60:546-554.
11. Mauch, T. J., T. M. Donohue, R. K. Zetterman. 1986. Covalent binding of acetaldehyde selectively inhibits the catalytic activity of lysine-dependent enzymes. Hepatology. 6:263-269.

12. Savolainen M., E. Baraona, and C. S. Lieber. 1987. Acetaldehyde binding increases the catabolism of rat serum low-density lipoprotein. Life Sci. 40:841846.

13. Israel, Y., E. Hurwitz, O. Niemelä, and R. Arnon. 1986. Monoclonal and polyclonal antibodies against acetaldehyde-containing epitopes in acetaldehydeprotein adducts. Proc. Natl. Acad. Sci. USA. 83:7923-7927.

14. Niemelä, O., F. Klajner, H. Orrego, E. Vidins, L. Blendis, and Y. Israel. 1987. Antibodies against acetaldehyde-modified protein epitopes in human alcoholics. Hepatology. 7:1210-1214.

15. Hoerner, M., U. J. Behrens, T. M. Worner, I. Blacksberg, R. Braley, F. Schaffner, and C. S. Lieber. 1988. The role of alcoholism and liver disease in the appearance of serum antibodies against acetaldehyde adducts. Hepatology. 8:569-574

16. Izumi, N., Y. Sakai, W. Koyama, and Y. Hasumura. 1989. Clinical significance of serum antibodies against alcohol-altered hepatocyte membrane in alcoholic liver disease. Alcohol. Clin. Exp. Res. 13:762-765.

17. Israel, Y., H. Orrego, and O. Niemelä. 1988. Immune responses to alcoho metabolites: pathogenic and diagnostic implications. Semin. Liver Dis. 8:81-90.

18. Lin, R. C., S. R. Smith, and L. Lumeng. 1988. Detection of a protein-acetaldehyde adduct in the liver of rats fed alcohol chronically. J. Clin. Invest. 81:615-619.

19. Lin, R. C., Lumeng, L., Shahidi, S., Kelly, T., and D. C. Pound. 1990 Protein-acetaldehyde adducts in serum of alcoholic patients. Alcohol. Clin. Exp. Res. 14:438-443.

20. Lin, R. C., and L. Lumeng. 1989. Further studies on the $37 \mathrm{kD}$ liver protein-acetaldehyde adduct that forms in vivo during chronic alcohol ingestion. Hepatology. 10:807-814.

21. Lin, R. C., Fillenwarth, M. J., Minter, R., and L. Lumeng 1990. Formation of $37-\mathrm{kD}$ protein-acetaldehyde adduct in primary cultured rat hepatocytes exposed to alcohol. Hepatology. 11:401-407.

22. Behrens, U. J., M. Hoerner, J. M. Lasker, and C. S. Lieber. 1988. Formation of acetaldehyde adducts with ethanol-inducible P450IIE 1 in vivo. Biochem Biophys. Res. Commun. 154:584-590.

23. Seppä, K., Sillanaukee, P., and T. Koivula. 1990. The efficiency of a questionnaire in detecting heavy drinkers. Br. J. Addict. 85:1639-1645.

24. Glanville, R. W. 1981. Preparation of type I and type III collagens, their pN-collagens and aminoterminal extension peptides. In Immunochemistry of the extracellular matrix. H. Furthmayr, editor. CRC Press, Boca Raton, FL 43-53.

25. Steinberger, L. A. 1974. Immunocytochemistry. Prentice-Hall, Eaglewood Cliffs, NJ. 129 pp.

26. Parkkila, S., K. Kaunisto, L. Rajaniemi, T. Kumpulainen, K. Jokinen, and H. Rajaniemi. 1990. Immunohistochemical localization of carbonic anhydrase isoenzymes VI, II, and I in human parotid and submandibular glands. $J$. Histochem. Cytochem. 38:941-947.

27. Nuutinen, H., K. Lindros, and M. Salaspuro. 1983. Determinants of blood acetaldehyde level during ethanol oxidation in chronic alcoholics. Alcohol. Clin. Exp. Res. 7:163-168.

28. Medina, V. A., T. M. Donohue, M. F. Sorrell, and D. J. Tuma. 1985. Covalent binding of acetaldehyde to hepatic proteins during ethanol oxidation. $J$. Lab. Clin. Med. 105:5-10.

29. Wickramasinghe, S. N., B. Gardner, and G. Barden. 1986. Cytotoxic protein molecules generated as a consequence of ethanol metabolism in vitro and in vivo. Lancet ii:823-826.

30. Yokoyama, H., H. Ishii, S. Nagata, S. Kato, A. Yokoyama, T. Takagi, M. Tsuchiya, and K. Kamegaya. 1990. Experimental hepatitis induced by ethanol after immunization with acetaldehyde adduct in guinea pig. Hepatology. 12:903a. (Abstr.)

31. Dole, V. P. 1986. On the relevance of animal models to alcoholism in humans. Alcohol. Clin. Exp. Res. 10:361-363.

32. Yokoyama, H., Ishii, H., Nagata, S., Kato, S., and M. Tsuchiya. 1990 Detection of acetaldehyde adducts in hepatic microsomes and cytosol after ethanol consumption. Alcohol. Clin. Exp. Res. 14:355a. (Abstr.)

33. Lieber, C. S. 1988. Biochemical and molecular basis of alcohol-induced injury to liver and other tissues. N. Engl. J. Med. 319:1639-1650.

34. Ingelman-Sundberg, M., I. Johansson, K. E. Penttilä, H. Glaumann, and K. O. Lindros. 1988. Centrilobular expression of ethanol-inducible cytochrome P-450 (IIE1) in rat liver. Biochem. Biophys. Res. Commun. 157:55-60.

35. Tsutsumi, M., J. M. Lasker, M. Shimizu, A. S. Rosman, and C. S. Lieber 1989. The intralobular distribution of ethanol inducible P450IIEl in rat and human liver. Hepatology. 10:437-446.

36. Buehler, R., M. Hess, and J. P. von Wartburg. 1982. Immunohistochemical localization of human liver alcohol dehydrogenase in liver tissue, cultured fibroblasts and HeLa cells. Am. J. Pathol. 108:89-99.

37. Yamaguchi, M., J. Potter, and E. Mezey. 1988. Lobular distribution of alcohol dehydrogenase in the rat liver. Hepatology. 8:243-247. 
38. Fraenkel-Conrat, H., and B. Singer. 1988. Nucleoside adducts are formed by cooperative reaction of acetaldehyde and alcohols: possible mechanism for the role of ethanol in carcinogenesis. Proc. Natl. Acad. Sci. USA. 85:3758-3761.

39. Behrens, U. J., X.-L. Ma, E. Baraona, and C. S. Lieber. 1989. Acetaldehyde-collagen adducts in $\mathrm{CCl}_{4}$-induced liver injury in rats. Hepatology. 10:608a. (Abstr.)

40. Ma, X.-L., Baraona, E., Hernandez-Munoz, R., and C. S. Lieber. 1989 High levels of acetaldehyde in non-alcoholic liver injury after threonine or ethanol administration. Hepatology. 10:933-940.

41. Peterson, C. M., and B. K. Scott. 1989. Studies of whole blood associated acetaldehyde as a marker for alcohol intake in mice. Alcohol. Clin. Exp. Res. 13:845-847.

42. Crabb, D. W. 1990. Biological markers for increased risk of alcoholism and for quantitation of alcohol consumption. J. Clin. Invest. 85:311-315.

43. Tuma, D. J., M. R. Newman, T. M. Donohue, and M. F. Sorrell. 1987. Covalent binding of acetaldehyde to proteins: participation of lysine residues. Alcohol. Clin. Exp. Res. 11:579-584.
44. Steinbrecher, U. P., M. Fisher, J. F. Witztum, and L. K. Curtiss. 1984. Immunogenicity of homologous low density lipoprotein after methylation, ethylation, acetylation, or carbamylation: generation of antibodies specific for the derivatized lysine. J. Lipid Res. 25:1109-1116.

45. Palinski, W., M. E. Rosenfeld, S. Yläherttuala, G. C. Gurtner, S. S. Socher, S. W. Butler, S. Parthasarathy, T. E. Carew, D. Steinberg, and J. L. Witztum. 1989. Low density lipoprotein undergoes oxidative modification in vivo. Proc. Natl. Acad. Sci. USA. 86:1372-1376.

46. San George, R. C., and H. D. Hoberman. 1986. Reaction of acetaldehyde with hemoglobin. J. Biol. Chem. 261:6811-6821.

47. Acharya, A. S., and J. M. Manning. 1983. Reaction of glycolaldehydes with proteins: latent crosslinking potential of $\alpha$-hydroxyaldehydes. Proc. Natl. Acad. Sci. USA. 80:3590-3594.

48. Fantl, W. J., L. R. Manning, H. Veno, A. Di Donato, and J. M. Manning. 1987. Properties of carboxymethylated cross-linked hemoglobin A. Biochemistry. 26:5755-5761 